\title{
Correction to: Mesenchymal stem cells decrease blood-brain barrier permeability in rats with severe acute pancreatitis
}

Ronggui Lin ${ }^{1 \dagger}$, Ming Li ${ }^{2 \dagger}$, Meiqin Luo ${ }^{3}$, Tianhong Teng ${ }^{1}$, Yu Pan ${ }^{1}$ and Heguang Huang ${ }^{1 *}$

\author{
* Correspondence: \\ Heguanghuang2@163.com \\ ${ }^{\dagger}$ Ronggui Lin and Ming Li \\ contributed equally to this work. \\ ${ }^{1}$ Department of General surgery, \\ Fujian Medical University Union \\ Hospital, 29 Xinquan Road, Fuzhou, \\ Fujian 350001, People's Republic of \\ China \\ Full list of author information is \\ available at the end of the article
}

\section{Correction to: Cell Mol Biol Lett (2019) 24:43 \\ https://doi.org/10.1186/s11658-019-0167-8}

Following publication of the original article [1], the author informed us that Fig. 5 was incorrect.

The correct figure is given below.

\section{Author details}

'Department of General surgery, Fujian Medical University Union Hospital, 29 Xinquan Road, Fuzhou, Fujian 350001, People's Republic of China. ${ }^{2}$ Department of Histology and Embryology, Hunan University of Medicine, Huaihua, Hunan, China. ${ }^{3}$ Department of Orthopedics, Fujian Medical University Union Hospital, Fuzhou, Fujian, China.

Published online: 26 August 2019

\section{Reference}

1. Lin R, et al. Mesenchymal stem cells decrease blood-brain barrier permeability in rats with severe acute pancreatitis. Cell Mol Biol Lett. 2019;24:43. https://doi.org/10.1186/s11658-019-0167-8. 


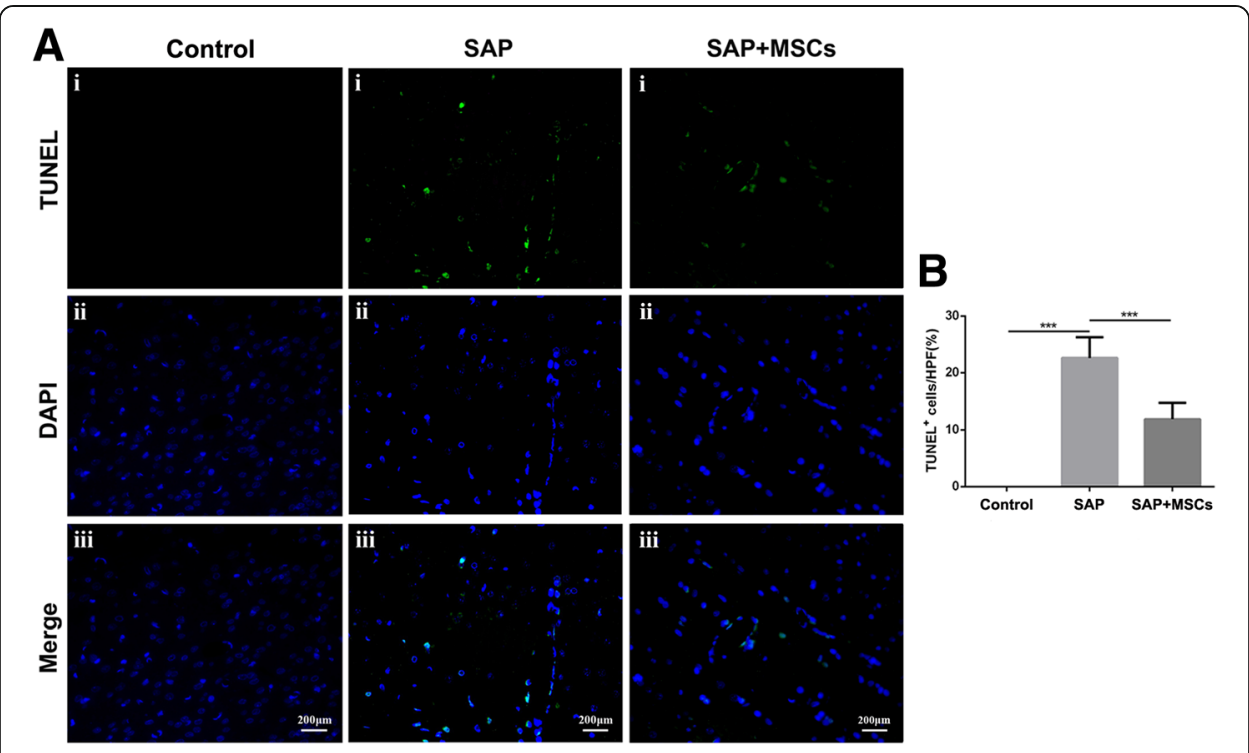

Fig. 5 MSCS reduced BMEC apoptosis in the brains of SAP rats. a TUNEL staining of the brain, scale bar $=200 \mu \mathrm{m}$. No apoptosis was observed in the control group, but numerous apoptotic cells, mainly BMECs, were observed in the SAP group. Fewer apoptotic cells were observed in the SAP+MSCs group than in the SAP group. b Statistical analysis of TUNEL-positive cells. ${ }^{* * *} p<0.001$ 